\title{
On Selection dynamics for competitive interactions
}

\author{
Pierre-Emmanuel Jabin \\ TOSCA project-team, INRIA Sophia Antipolis - Méditerranée, \\ 2004 rte des Lucioles, BP. 93, 06902 Sophia Antipolis Cedex, France \\ Lab. Dieudonné, Université de Nice Sophia-Antipolis, \\ Parc Valrose - 06108 Nice Cedex 02,France. \\ e-mail: jabin@unice.fr \\ Gaël Raoul \\ CMLA, ENS Cachan, CNRS, PRES UniverSud, \\ 61, avenue du Président Wilson, 94235 Cachan Cedex, France \\ e-mail: raoul@cmla.ens-cachan.fr
}

\begin{abstract}
In this paper, we are interested in an integro-differential model that describe the evolution of a population structured with respect to a continuous trait. Under some assumption, we are able to find an entropy for the system, and show that some steady solutions are globaly stable. The stability conditions we find are coherent with those of Adaptive Dynamics.
\end{abstract}

AMS class. No: 35B40, 92D15

\section{Introduction}

\subsection{The model and its basic properties}

We are interested in the dynamics of a population of individuals with a quantitative trait. The reproduction rate of each individual is determined by its trait and the environment, leading therefore to selection.

The environment itself is influenced by the population and the corresponding feedback can make the possible asymptotic behaviour quite complex. However we limit ourselves to a class of competitive interactions that will ensure the convergence to a unique limit. 
We denote by $f(t, x)$ the density of individuals with trait $x$. The space of traits $X$ can be fairly general, even though for simplicity we will take a subset of $\mathbb{R}^{d}$. We assume that $f$ satisfies the following equation:

$$
\left\{\begin{array}{l}
\partial_{t} f(t, x)=\left(a(x)-\int b(x, y) f(t, y) d y\right) f(t, x), \text { for } t \geq 0, x \in X \\
f(0, x)=f^{0}(x)>0 \text { for } x \in X .
\end{array}\right.
$$

The term $a(x)-\int b(x, y) f(t, y) d y$ is the reproduction rate and it takes into account the effect that the population itself has on the environment through the integral kernel. Competition usually means taking $b$ nonnegative (but we will need stronger and more precise assumptions below). A general discussion on this model as well as an existence proof can be found in [7]. Our goal is mainly to precise the analysis of the asymptotic behaviour that was initiated in this article.

Equation (1.1) corresponds to models frequently used, see for instance (among many) [3], [11], [15], [22]. It can be derived from stochastic models of finite populations (see [6] or [12]). Note that the environment is assumed to react instantaneously to the population, and in many cases it would be more realistic to also write down an evolution equation on some environmental variables (see an example in [1]). This would unfortunately not always fit with the framework developed here.

Other changes (not necessarily compatible with our analysis) include spatial effects (see for instance [14], [24]), random environments ([23] for example), and of course non competitive interactions should lead to quite different asymptotics (see [13] for a study of mutualism).

We will often denote

$$
b[f](x)=\int_{X} b(x, y) f(y) d y
$$

and (with a slight abuse of notation) if $f$ is a Radon measure, we will write $f(x) d x$ instead of the correct $d f(x)$.

Existence of regular (Lipschitz for instance) or measure valued solutions to (1.1) is not difficult, provided that the coefficients have enough regularity. We assume that

$$
a \in W^{1, \infty}(X), \quad b \in W^{1, \infty}(X) .
$$

Under assumption (1.2), there exists a unique $f \in W^{1, \infty}\left([0, T], W^{1, \infty}(X)\right)$ (or, depending on the smoothness of $f^{0}, W^{1, \infty}\left([0, T], M^{1}(X)\right)$ ), solution to (1.1) on any time interval $[0, T]$.

We are of course interested in the long time behaviour of this solution. The first point is to make sure that the total population $\int_{X} f(t, x) d x$ remains 
bounded from below and above. This is ensured (see [7]) by the following hypothesis:

$$
\mathcal{O}:=\{x \in X ; a(x)>0\} \neq \emptyset, \quad \inf _{x, y \in X} b(x, y)>0 .
$$

This assumption can be somewhat relaxed (in particular if $X$ is not compact, for example $X=\mathbb{R}$ ).

Of course in a general framework (and even with a non vanishing population) there is no particular reason to expect that the solution has a limit. However we only consider competitive interaction between individuals: The individual with trait $y$ only has a negative effect on the one with trait $x$ because of the positivity assumption on $b$ in (1.3).

It is widely believed that competition will induce a convergence to the "optimal" repartition of traits. Following ideas already well developed in adaptive dynamics (see [8] for instance), we introduce the notion of Evolutionary Stable Distribution, which is a global version of the ESS used in Adaptive Dynamics (see [7]):

Definition 1 The measure $\bar{f} \in M^{1}(\mathbb{R})$ is called an Evolutionary Stable Distribution (ESD) for the equation (1.1) if:

$$
\left\{\begin{array}{l}
\forall x \in \operatorname{supp} \bar{f}, \quad 0=a(x)-\int b(x, y) \bar{f}(y) d y \\
\forall x \in X, \quad 0 \geq a(x)-\int b(x, y) \bar{f}(y) d y
\end{array}\right.
$$

It seems that the ESD is generically a sum of Dirac masses

$$
\bar{f}=\sum_{i=1}^{n} \alpha_{i} \delta_{x_{i}},
$$

which can be interpreted as a mode of speciation: The population which was initially dispersed along all the traits, now concentrates along a few of them ("species"). Nevertheless the ESD may sometimes be a continuous function, as in one of the examples shown later in this article.

The existence of an ESD is not always easy (see [21]), and it can require more assumptions on $X$ than those we have made (closeness for instance). In order to remain fairly general, we therefore simply assume that one has at least one ESD:

$$
\exists \bar{f} \in M^{1}(X), \quad \bar{f} \geq 0, \quad \bar{f} \text { satisfying (1.4). }
$$

The ESD is however not necessarily unique (see [20]), thus making convergence for any initial data doubtful. 
There have already been many studies of existence and stability of the steady-states for this kind of models, we refer for example to [4], [9], [17], etc. The novelty of our result is that we give a general additional condition on $b$ which ensures the convergence toward the ESS.

Let us also mention that this kind of results may be seen as a first step toward a rigorous mathematical study of selection/mutation models. In particular, when there is a time scale separation between the two phenomena, the approach followed in [10] leads to a model like

$$
\partial_{t} f_{\varepsilon}=\frac{1}{\varepsilon}\left(a-b\left[f_{\varepsilon}\right]\right) f_{\varepsilon}+K_{\varepsilon}\left[f_{\varepsilon}\right],
$$

with $K_{\varepsilon}$ a mutation kernel and $\varepsilon$, equal to the ratio of the time scales, tending to 0 .

At each time, one then expects $f_{\varepsilon}$ to be at equilibrium (or ESD) w.r.t. the fast dynamics (which exactly corresponds to the long time asymptotics of (1.1)). This fast dynamics equilibrium would be taken among all traits present at that time (for which $f_{\varepsilon}$ is not too small). Therefore the ESD should evolve in time as new traits are added, thanks to the mutations.

In the case of one dominant trait, one would expect to essentially recover the canonical equation of adaptive dynamics (see [5] for another approach). But our approach has the additional advantage of not requiring that only one trait be present; It is then possible to see branching occur for instance.

The analysis in [10] was essentially formal (see [2] for a rigorous result with additional assumptions and [19] for a related derivation). A better understanding of the selection mechanism appears necessary to go further.

The set $X$ is typically a subset of $\mathbb{R}^{d}$ but not necessarily a regular one. Considering (1.1) alone, it would be natural to take a ball for $X$ or any other nice domain. However many applications demand more singular sets. For instance the derivation from (1.7) would typically lead to $X$ being the set of points where some function is larger than a given value.

Instead of a regularity assumption, each point is assumed to have a non vanishing density with respect to the Lebesgue measure:

$$
\inf _{x \in X} \inf _{0<r<1} r^{-d} \int_{X} \mathbb{I}_{|x-y| \leq r} d y>0 .
$$

Of course the Lebesgue measure is used only for convenience and could be replaced by any other $\sigma$-finite Radon measure as long as (1.8) is satisfied for some positive $d$ : Restrictions of Hausdorff measures for example.

With some effort, the theory presented here could even be generalized to a complete metric set $X$ endowed with a measure satisfying adequate regularity properties, and (1.8) with any function dominated by the exponential 
replacing $r^{d}$. Note finally that some closeness or compactness assumption on $X$ is typically required in order to get the existence of a stable equilibrium to $(1.1)$, see [21].

\subsection{The results}

We introduce a stronger version of the competition assumption, namely

$$
\forall g \in M^{1}(X) \backslash\{0\}, \quad \iint b(x, y) g(x) g(y) d x d y>0 .
$$

Note that (1.9) is automatically satisfied for $g \geq 0$ because of Assumption (1.3). As there is no sign condition on $g$ in (1.9), it is indeed stronger than $(1.3)$.

One can check using classical methods that $b$ defines then a scalar product over $M^{1}(X)$ :

Proposition 1 Assume that $b \in L^{\infty}(X \times X)$ satisfies (1.9). Then we can define a scalar product over $M^{1}(X)$ and the corresponding norm by

$$
\begin{aligned}
\langle g, h\rangle_{b} & =\frac{1}{2} \iint b(x, y)(g(x) h(y)+h(x) g(y)) d x d y, \\
\|g\|_{b} & =\left(\iint b(x, y) g(x) g(y) d x d y\right)^{\frac{1}{2}} .
\end{aligned}
$$

Remark 0.1 The topology induced by this norm is weaker than the usual topology on measures. For example, in the case when $X:=\mathbb{R}, b(x, y):=$ $e^{-|x-y|^{2}}$, if $\left\|f_{n}\right\|_{b} \rightarrow 0$, then

$$
\int \phi(x) f_{n}(x) d x \rightarrow 0
$$

for all $\phi \in L^{2}(\mathbb{R})$ such that $\xi \in \mathbb{R} \mapsto e^{\xi^{2}} \mathcal{F}(\phi)(\xi) \in L^{2}(\mathbb{R})$.

The strong competition assumption (1.9) is directly connected to the stability of the ESD (and the CSS condition of adaptive dynamics). It does not seem to be related however to a condition on the spectrum of a linearization of the equation near the equilibrium $\bar{f}$. Under assumption (1.9), $\bar{f}$ is unique:

Theorem 1 Assume (1.2), (1.3), (1.9). Then there exists at most one $\bar{f} \in$ $M^{1}(X)$ satisfying Def. (1.4). 
Remark 1.1 As seen in the proof in subsection 2.1, assumption (1.9) may be replaced by a slighty different version

$$
a(x)-b[\bar{f}](x)<0 \quad \forall x \notin \operatorname{supp} \bar{f},
$$

and

$$
\begin{array}{ll}
\int_{X^{2}} b(x, y) g(x) g(y) d x d y>0 & \forall g \in M^{1}(X) \backslash\{0\} \text { with supp } \subset \subset \operatorname{supp} \bar{f} \\
\int_{X^{2}} b(x, y) g(x) g(y) d x d y \geq 0 & \forall g \in M^{1}(X) .
\end{array}
$$

It is however usually more complicated to check this alternative assumption, since it requires the computation of $\bar{f}$.

Eq. (1.1) now has a Lyapunov functional

$$
F(t)=\int_{X}\left[\bar{f}(x) \log \left(\frac{\bar{f}(x)}{f(t, x)}\right)+f(t, x)-\bar{f}(x)\right] d x
$$

and thanks to that we can show the convergence of $f(t, \cdot)$ towards $\bar{f}$ :

Theorem 2 Assume (1.2), (1.3), (1.6) and (1.9). Then if $f^{0} \in M^{1}(X)$ is such that $F(0)<\infty$ (with $F$ defined by (1.14)), $f(t, \cdot)$ converges to $\bar{f}$ as $t \rightarrow \infty$ in the sense that:

$$
\begin{gathered}
\|f(t, \cdot)-\bar{f}(\cdot)\|_{b} \rightarrow 0, \\
\|f(t, \cdot)\|_{L^{1}(|a(x)-b[\bar{f}](x)| d x)} \rightarrow 0 .
\end{gathered}
$$

Remark 2.1 If b satisfies (1.12) and (1.13) instead of (1.9) in Thm 2, $\|\cdot\|_{b}$ does not define a norme anymore, but the estimates (1.15) and (1.16) still hold.

Theorem 2 is not satisfactory in many situations. Indeed if we take $f^{0}$ bounded from below and above, then $F(0)$ is bounded if and only if

$$
\int_{X} \bar{f} \log \bar{f}<\infty
$$

This requires $\bar{f} \in L \log L$ and in particular $\bar{f} \in L^{1}$. Therefore we cannot use Thm 2 when $\bar{f}$ is only a measure, for instance a sum of Dirac masses. 
Unfortunately, it is believed to be the most "common" form of $\bar{f}$ (remember that one expects speciation).

Obtaining convergence in this singular situation is more complicated and we are able to do it only when some additional symmetry is available on $b$, namely when

$$
\begin{aligned}
& \exists M, N \in L^{\infty}(X), \quad M, N>0, \quad \inf _{x} \frac{M(x)}{M(x)+N(x)}>0, \\
& \forall x, y \in X, \quad b(x, y) M(x)=b(y, x) N(y) .
\end{aligned}
$$

If $M, N \neq 1$, then the natural bilinear form associated to $b$ is not any more given by (1.10), and one replaces assumption (1.9) by

$$
\int_{X^{2}} b(x, y) g(x) g(y) M(x) d x d y>0 \quad \forall g \in M^{1}(X), g \neq 0 .
$$

Finally we need to assume that $f^{0}$ does not vanish on supp $\bar{f}$ (this was included in the assumption $F(0)<\infty$ in Thm. 2). We suppose therefore that

$$
\liminf _{r \rightarrow 0} \inf _{\omega_{r}} f^{0}>0, \quad \text { where } \quad \omega_{r}=\{x \in X, d(x, \operatorname{supp} \bar{f})<r\} .
$$

This sometimes has to be relaxed, if for instance supp $\bar{f}$ is not compact (the previous assumption is then incompatible with $\left.f^{0} \in L^{1}\right)$. One therefore introduces the weaker assumption that there exist $r_{0}>0$ and $C>0$ such that

$$
\sup _{r \geq r_{0}} \int_{X} \bar{f} \log \frac{1}{f_{r}^{0}(x)} d x<C, \quad \text { where } f_{r}^{0}(x)=r^{d} \int_{X} f^{0}(y) e^{-r|x-y|} d y .
$$

We may now state our main result:

Theorem 3 Assume (1.8), (1.2), (1.3), (1.6), (1.17) and (1.18). Consider any $f^{0} \in L^{1}(X) \cap L^{\infty}($ supp $\bar{f})$ with (1.19) or (1.20). The corresponding solution $f(t, \cdot)$ to (1.1) converges to $\bar{f}$ as $t \rightarrow \infty$ in the sense that:

$$
\begin{gathered}
\|f(t, \cdot)-\bar{f}(\cdot)\|_{b M}=O\left(\frac{\log t}{t}\right), \\
\|f(t, \cdot)\|_{L^{1}[|a(x)-b[\bar{f}](x)|(M(x)+N(x)) d x]}=O\left(\frac{\log t}{t}\right) .
\end{gathered}
$$


Remark 3.1 If b satisfies (1.12), and (1.13) with $\tilde{b}:=b M$ instead of (1.18) in Thm 3, $\|\cdot\|_{\tilde{b}}$ does not define a norm anymore, but the estimates (1.21) and (1.22) still hold.

Remark 3.2 If $\bar{f}$ is singular (a sum of Dirac masses for example) then the rates of convergence given in (1.21), (1.22) are very close to being optimal. Indeed the sum of the two expressions estimated in Thm 3 is exactly the dissipation rate of the modified functional

$$
\int[-\bar{f}(x) \log (f(t, x))+f(t, x)-\bar{f}(x)] M(x) d x .
$$

When $\bar{f}$ is singular and $f(t, \cdot) \rightarrow \bar{f}$, then this functional has to converge to $-\infty$ and therefore its dissipation rate cannot be integrable in time. As a consequence, one cannot have

$$
\|f-\bar{f}\|_{b M}^{2}+\|f\|_{L^{1}(|a-b[\bar{f}]|(M+N) d x)}=O\left(t^{-\alpha}\right),
$$

with any $\alpha>1$. In particular exponential convergence cannot hold: the natural idea of linearizing the equation around the equilibrium in order to study convergence is most certainly very difficult to implement.

Remark 3.3 Assumption (1.19) and (1.8) could both be replaced by

$$
\inf _{\operatorname{supp} \bar{f}} \tilde{M} f^{0}(x)>0
$$

where $\tilde{M} f^{0}$ is a sort of maximal function of $f^{0}$ ("minimal" in fact) computed at $x$

$$
\tilde{M} f^{0}(x)=\inf _{r \rightarrow 0} \frac{1}{r^{d}} \int_{y \in X,|y-x|<r} f^{0}(y) d y .
$$

This is however not really an improvement, since it is not possible to have (1.23) for a bounded $f^{0}$ unless $X$ verifies (1.8) at least on supp $\bar{f}$.

Note also that (1.19) is trivially satisfied provided that in $f_{\text {supp } \bar{f}} f^{0}>0$ and that $f^{0}$ is uniformly continuous on supp $\bar{f}$.

An important consequence of those convergence results is the stability of ESD with respect to perturbations of the coefficients $a$ and $b$ :

Corollary 3.1 Assume that $X$ satisfies (1.8), $a_{1}, b_{1}$ and $a_{2}, b_{2}$ satisfy (1.2), (1.3), (1.6). We denote by $\bar{f}_{1}$ the ESD associated to $a_{1}, b_{1}$ and by $\bar{f}_{2}$ an ESD associated to $a_{2}, b_{2}$. 
- If $\bar{f}_{1} \in \operatorname{Llog} L \cap L^{1}(X)$ and (1.9) holds for $b_{1}$, then

$$
\left\|\bar{f}_{1}(\cdot)-\bar{f}_{2}(\cdot)\right\|_{b_{1}} \leq X\left(\left\|a_{1}-a_{2}\right\|_{\infty}+\mid b_{1}-b_{2} \|_{\infty}\right) .
$$

- If $b_{1}$ satisfies (1.17), (1.18) and

$$
\int_{X} \bar{f}_{1}(x) \log |x| d x<\infty
$$

then

$$
\left\|\bar{f}_{1}(\cdot)-\bar{f}_{2}(\cdot)\right\|_{b_{1} M} \leq X\left(\left\|a_{1}-a_{2}\right\|_{\infty}+\mid b_{1}-b_{2} \|_{\infty}\right)
$$

\subsection{Examples of application}

We first introduce a well-known example of a continuous ESD, in which $a, b$ are Gaussian functions. Numerical illustrations of this example can be found in [7].

Example 3.1 Consider the case of Gaussian coefficients a and b:

$$
\begin{aligned}
& X:=\mathbb{R}, \quad a(x):=\frac{1}{\sqrt{2 \pi}\left(\gamma_{1}+\gamma_{2}\right)} e^{\frac{-x^{2}}{2\left(\gamma_{1}+\gamma_{2}\right)^{2}}}, \\
& b(x, y):=B(x-y)=\frac{1}{\sqrt{2 \pi} \gamma_{1}} e^{\frac{-(x-y)^{2}}{2 \gamma_{1}^{2}}},
\end{aligned}
$$

where $\gamma_{1}, \gamma_{2}>0$. Then for any initial condition $f^{0} \in L^{1}\left(e^{\frac{-x^{2}}{2 \gamma_{2}^{2}}} d x\right), f^{0}>0$, the solution $f(t, \cdot)$ to (1.1) will converge for the norm $\|\cdot\|_{b}$ when $t \rightarrow \infty$ to the ESD:

$$
\bar{f}(x):=\frac{1}{\sqrt{2 \pi} \gamma_{2}} e^{\frac{-x^{2}}{2 \gamma_{2}^{2}}} .
$$

Moreover, if $\tilde{a}, \tilde{b}$ satisfying (1.2), (1.3) are close enough to $a, b$ in $L^{\infty}(\mathbb{R})$, then any ESD $\tilde{f}$ associated to $\tilde{a}, \tilde{b}$ will be close to $\bar{f}$ in the sense that:

$$
\|\tilde{f}-\bar{f}\|_{b} \leq O\left(\|\tilde{a}-a\|_{\infty}+\mid \tilde{b}-b \|_{\infty}\right) .
$$

Finally, if $\tilde{b}$ satisfies (1.9), this ESD is unique and global attractor for the norm $\|f\|_{\tilde{b}}$. 
The coefficients $a$ and $b$ defined by (1.26) have no real biological justification (see [1]), but they have been widely used in theoretical biology (see $[22])$. The stability of $\bar{f}$, and in particular the stability of $\bar{f}$ with respect to perturbations of the coefficients $a, b$ has been discussed for instance in [11], [17], [7]. In [17], it is shown that for arbitrary small perturbations of $a$ and $b$, only discrete ESD can exist. There is no contradiction with our result, since $\bar{f}$ is only stable in a very weak topology, with respect to perturbations of the coefficients. This nevertheless means that the continuous ESD $\bar{f}$ given by (1.27) may indeed be observed (though approximated by Dirac masses).

Proof of Example 3.1. As $\mathcal{F} B>0$ (where $\mathcal{F}$ denotes the Fourier transform), one can consider $K:=\mathcal{F}^{-1}(\sqrt{\mathcal{F} B})$. Then, for any $g \in M^{1}(\mathbb{R}), g \neq 0$,

$$
\begin{aligned}
\int_{\mathbb{R}^{2}} b(x, y) g(x) g(y) d x d y & =\int_{\mathbb{R}} g(x) B *_{x} g(x) d x \\
& =\int_{\mathbb{R}} g(x) K *_{x} K *_{x} g(x) d x \\
& =\int_{\mathbb{R}}\left(K *_{x} g(x)\right)^{2} d x \\
& >0,
\end{aligned}
$$

that is $b$ satisfies the assumption (1.9), and Thm 1 and Thm 2 apply, wich shows the first part of the example. Cor. 3.1 implies (1.28) (for the existence of such an ESD, see [21]). If $\tilde{b}$ satisfies (1.9), one can apply Thm 1 to show that there exists at most one $\operatorname{ESD} \tilde{f}$, and apply Thm 3 to obtain that $\tilde{f}$ is a global attractor, which completes the proof.

The next example assumes that the individuals interact through the consumption of a finite number $n$ of ressources:

Example 3.2 Consider the case where $a \in W^{1, \infty}(X)$, and

$$
b(x, y):=\sum_{i=1}^{n} \eta_{i}(x) \eta_{i}(y) K(y)
$$

where $\eta_{1}, \ldots, \eta_{n}, K \in W^{1, \infty}(X)$, and $K>0$. It can be used to model a population that has access to $n$ different types of ressources, the efficiency at which an individual of trait $x$ eats the ressource $i$ is then $\eta_{i}(x)^{-1}$.

Let $\bar{f}=\sum_{i=1}^{n} \bar{\rho}_{i} \delta_{\bar{x}_{i}}$ be an ESD. If:

$$
a(x)-\int_{X} b(x, y) \bar{f}(y) d y=a(x)-\sum_{i=1}^{n} b\left(x, \bar{x}_{i}\right) \bar{\rho}_{i}<0, \quad \forall x \neq \bar{x}_{1}, \ldots, \bar{x}_{n},
$$

$$
\left(\eta_{i}\left(\bar{x}_{j}\right)\right)_{i, j=1, \ldots, n} \text { is non-singular, }
$$


then for any initial data $f^{0}>0$ satisfying (1.19), $f$ will converge to $\bar{f}$.

Proof of Example 3.2 We show that conditions (1.12), (1.13) hold with $\tilde{b}(x, y):=K(x) b(x, y)$. If $g \in M^{1}(X)$,

$$
\begin{aligned}
\int_{X^{2}} \tilde{b}(x, y) g(x) g(y) d x d y & =\sum_{i=1}^{n}\left(\int_{X} \eta_{i}(x) K(x) g(x) d x\right)^{2} \\
& \geq 0 .
\end{aligned}
$$

if $g \in M^{1}(X), g \neq 0$, and $\operatorname{supp} g \subset \operatorname{supp} \bar{f}$, then $g(x)=\sum_{i=1}^{n} r_{i} \delta_{\bar{x}_{i}}$, and:

$$
\begin{aligned}
\int_{X^{2}} \tilde{b}(x, y) g(x) g(y) d x d y & =\sum_{i=1}^{n}\left(\sum_{j=1}^{n} r_{j} \eta_{i}\left(\bar{x}_{j}\right) K\left(\bar{x}_{j}\right)\right)^{2} \\
& =\left\|\left(\eta_{i}\left(\bar{x}_{j}\right)\right)_{i, j=1, \ldots, n}\left(K\left(\bar{x}_{i}\right) r_{i}\right)_{i=1, \ldots, n}\right\|_{2}^{2} \\
& >0,
\end{aligned}
$$

since $\left(\eta_{i}\left(\bar{x}_{j}\right)\right)_{i, j=1, \ldots, n}$ is invertible (1.31) and $K>0$.

Since (1.32), (1.33) and (1.30) are satisfied, conditions (1.12) (1.13) hold, and Thm 1 apply. Since $K(x) b(x, y)=b(y, x) K(y),(1.17)$ is satisfied with $M(x):=K(x)$ and thus Rem 1.2 apply, wich proves the assertion of the example. Notice that $b$ cannot satisfy (1.18) as by taking $g$ orthogonal to $\eta_{1} K, \ldots, \eta_{n} K$ in $L^{2}(X),(1.9)$ does not hold.

Remark 3.4 More complicated interactions are commonly considered like a chemostat

$$
\frac{d}{d t} I_{k}=-S_{k}+\delta_{k} I_{k}+\gamma_{k} \int_{X} \mu_{k}(x) f(t, x) d x .
$$

Those do not fit in the simple framework developed here and for this reason we stick to (1.29).

\section{Proofs of the results}

\subsection{Proof of Theorem 1}

We argue by contradiction and therefore assume that one has two measures $\bar{f}_{1}$ and $\bar{f}_{2}$ satisfying the ESD definition (1.4). Note that since $a-b\left[\bar{f}_{i}\right] \leq 0$ for all $i$ and $\bar{f}_{i} \geq 0$, one has

$$
\int_{X}\left(a-b\left[\bar{f}_{1}\right]\right) \bar{f}_{2} d x+\int_{X}\left(a-b\left[\bar{f}_{2}\right]\right) \bar{f}_{1} d x \leq 0 .
$$


On the other hand, since $a-b\left[\bar{f}_{i}\right]=0$ on the support of $\bar{f}_{i}$,

$$
\int_{X}\left(a-b\left[\bar{f}_{i}\right]\right) \bar{f}_{i} d x=0
$$

One can also observe that

$$
\begin{aligned}
\int_{X}\left(a-b\left[\bar{f}_{1}\right]\right) \bar{f}_{2} d x+ & \int_{X}\left(a-b\left[\bar{f}_{2}\right]\right) \bar{f}_{1} d x=\int_{X} b\left[\bar{f}_{2}-\bar{f}_{1}\right] \bar{f}_{2} d x \\
& +\int_{X} b\left[\bar{f}_{1}-\bar{f}_{2}\right] \bar{f}_{1} d x \\
= & \int_{X^{2}}\left(\bar{f}_{2}(x)-\bar{f}_{1}(x)\right) b(x, y)\left(\bar{f}_{2}(y)-\bar{f}_{1}(y)\right) d x d y
\end{aligned}
$$

Thanks to assumption (1.9), this last integral is nonnegative and therefore vanishes (it also has to be nonpositive). This implies $\bar{f}_{1}=\bar{f}_{2}$ (still thanks to $(1.9))$.

\subsection{Proof of Theorem 2}

Step 1: We show that $F$ is decreasing.

We define the functional $F$ by (1.14) and also consider the regularized version

$$
F_{n}(t)=\int_{X}\left[\bar{f}_{n}(x) \log \left(\frac{\bar{f}_{n}(x)}{f(t, x)}\right)+\left(f(t, x)-\bar{f}_{n}(x)\right)\right] d x
$$

with $\bar{f}_{n}$ a regularization of $\bar{f}$ (through convolution for instance). As $F(0)<$ $\infty, F_{n}(0)$ is uniformly bounded. Moreover

$$
\begin{aligned}
\frac{d}{d t} F_{n}(t)= & -\int_{X^{2}} b(x, y)\left(f(t, x)-\bar{f}_{n}(x)\right)\left(f(t, y)-\bar{f}_{n}(y)\right) d x d y \\
& +\int_{X}(a(x)-b[\bar{f}]) f(t, x) d x+R_{n}
\end{aligned}
$$

with

$$
R_{n}=\int_{X} b\left[\bar{f}-\bar{f}_{n}\right] f d x-\int_{X}\left(a-b\left[\bar{f}_{n}\right]\right) \bar{f}_{n} d x .
$$

The quantity $R_{n}$ converges toward 0 as $n \rightarrow+\infty$ uniformly w.r. to $t \in \mathbb{R}_{+}$, since $\bar{f}_{n} \rightarrow \bar{f}$, and since the definition (1.4) implies that

$$
\int_{X}(a-b[\bar{f}]) \bar{f} d x=0 .
$$


Thanks to condition (1.9) on $b, d F_{n} / d t \leq R_{n}$, and so $F_{n}(t)$ is uniformly bounded in $n$ for a given $t>0$. Therefore $F(t)<\infty$ for all time, and in addition

$$
\begin{aligned}
\frac{d}{d t} F(t)= & -\int_{X^{2}} b(x, y)(f(t, x)-\bar{f}(x))(f(t, y)-\bar{f}(y)) d x d y \\
& +\int_{X}(a(x)-b[\bar{f}]) f(t, x) d x:=-D(f, \bar{f}) \leq 0
\end{aligned}
$$

It means that $t \mapsto F(t)$ is strictly decreasing as long as $f(t, \cdot) \neq \bar{f}$, and bounded from below by 0 . It follows that $F$ converges to a limit denoted by $\bar{F}$.

Step 2: We show that $\frac{d}{d t} F(t) \rightarrow 0$.

We first observe that $\frac{d}{d t} F(t)$ is Lipschitz continuous:

$$
\begin{aligned}
\left|\frac{d}{d t} \frac{d}{d t} F(t)\right| & \leq 2\|b\|_{\infty}\left\|\frac{d}{d t}(f-\bar{f})\right\|_{M^{1}}\|f-\bar{f}\|_{M^{1}} \\
& +\left(\|a\|_{\infty}+\|b\|_{\infty}\|\bar{f}\|_{M^{1}}\right)\left\|\frac{d}{d t} f\right\|_{M^{1}} \\
\leq & C_{F}<\infty,
\end{aligned}
$$

since $\left\|\frac{d}{d t} f\right\|_{M^{1}} \leq\left(\|a\|_{\infty}+\|b\|_{\infty}\|f\|_{M^{1}}\right)\|f\|_{M^{1}}$ and $f \in L^{\infty}\left(\mathbb{R}, M^{1}\right)$ (see [7]).

Let $\varepsilon>0$. Since $F(t) \rightarrow \bar{F}$, there exists $T>0$ such that $F(T) \leq \bar{F}+\varepsilon$, that is:

$$
\int_{T}^{\infty} \frac{d}{d t} F \geq-\varepsilon
$$

But since $\frac{d}{d t} F$ is Lipschitz, for any $t \geq T$ and $\tau \geq t$,

$$
\frac{d}{d t} F(\tau)<\frac{d}{d t} F(t)+C_{F}(\tau-t)
$$

and then:

$$
\int_{T}^{\infty} \frac{d}{d t} F \leq \int_{t}^{t+\frac{1}{2 C_{F}}\left|\frac{d}{d t} F(t)\right|} \frac{d}{d t} F \leq \frac{-1}{4 C_{F}}\left(\frac{d}{d t} F(t)\right)^{2} .
$$

Combining this with (2.1), we obtain:

$$
\forall t \geq T, \quad\left|\frac{d}{d t} F(t)\right| \leq 2 \sqrt{C_{F} \varepsilon}
$$

and thus $\frac{d}{d t} F(t) \rightarrow 0$. Finally, $D(f(t, \cdot), \bar{f}) \rightarrow 0$ implies that $\|f(t, \cdot)-\bar{f}\|_{b} \rightarrow 0$ and that $\|f(t, \cdot)\|_{L^{1}(|a(x)-b[\bar{f}](x)| d x)} \rightarrow 0$. 


\subsection{Proof of Theorem 3}

In the general case, when $\bar{f} \in M^{1}$ but $\bar{f}$ is not necessarly a function, the functional (1.14) is not finite. Nevertheless, it is possible to define instead

$$
\tilde{F}(t):=\int_{X}\left[\bar{f}(x) \log \left(\frac{1}{f(t, x)}\right)+(f(t, x)-\bar{f}(x))\right] M(x) d x .
$$

A regularization argument like the one used in the previous subsection shows that $\tilde{F}$ is finite at all times and strictly decreasing as long as $f \neq \bar{f}$. Unfortunately, this functional is not bounded from below anymore and this is not enough to conclude.

We show in a first step that in fact, the new functional $\tilde{F}$ does not decrease too fast. In a second step, we prove that the dissipation rate $D=-\frac{d \tilde{F}}{d t}$ can be controlled from above by a decreasing function $G$ (defined in (2.7)). We then combine the two previous estimates.

Step 1: Lower bound on $\tilde{F}$.

As $a$ and $b$ are Lipschitz-continuous, so is $a-b[f]$ w.r.t. $x$, and since $f:=$ $f(t, \cdot)$ is bounded in $L^{1}(X)$, there exists a constant $C$ independant of $t \in \mathbb{R}_{+}$ such that

$$
\forall x, y \in X, \quad a(y)-b[f](y) \geq a(x)-b[f](x)-C|x-y| .
$$

Now let us start by giving an upper bound on $f(t, x)$

$$
\begin{aligned}
\int_{X} f(t, y) d y & =\int_{X} f^{0}(y) e^{\int_{0}^{t}\left(a(y)-\int b(y, z) f(s, z) d z\right) d s} d y \\
& \geq \int_{X} \frac{f^{0}(y)}{f^{0}(x)}\left(f^{0}(x) e^{\int_{0}^{t}\left(a(x)-\int b(x, z) f(s, z) d z\right) d s}\right) e^{-C t|x-y|} d y \\
& \geq \frac{f(t, x)}{f^{0}(x)} \int_{X} f^{0}(y) e^{-C t|x-y|} d y
\end{aligned}
$$

As $t \rightarrow+\infty$, one finds thanks to the lower bound on $f^{0}(1.19)$ :

$$
\begin{aligned}
\inf _{x \in \operatorname{supp} \bar{f}} \int_{X} f^{0}(y) e^{-C t|x-y|} d y & \geq C \inf _{x \in \omega_{1 / t}} f^{0}(x) \int_{X} \mathbb{I}_{|y-x| \leq 1 / t} d y \\
& \geq C \inf _{x \in \omega_{1 / t}} \int_{X} \mathbb{I}_{|y-x| \leq 1 / t} d y .
\end{aligned}
$$

Using finally assumption (1.8) on the density of points of $X$,

$$
\inf _{x \in \operatorname{Supp}(\bar{f})} \int_{X} f^{0}(y) e^{-C t|x-y|} d y \geq C t^{-d} .
$$


If $f^{0}$ satisfies (1.19), then, combining (2.3) and (2.4), one gets:

$$
f(t, x) \leq C t^{d}, \quad \forall x \in \operatorname{supp} \bar{f} .
$$

This directly controls $\tilde{F}$ from below:

$$
\begin{aligned}
\tilde{F}(t) & =\int_{X}\left[\bar{f}(x) \log \left(\frac{1}{f(t, x)}\right)+(f(t, x)-\bar{f}(x))\right] M(x) d x \\
& \geq-C-\int \bar{f}(x) \log (f(t, x)) M(x) d x \\
& \geq-C-C \int \bar{f}(x) \log \left(t^{d}\right) M(x) d x \\
& \geq-C-C \log (t)
\end{aligned}
$$

where $C$ is still a constant (wich can be expressed in terms of the total mass $\left.\int_{X} f(t, \cdot) d x\right)$.

If, instead of (1.19), one takes (1.20), the estimate is almost the same. First, for any $x \in \operatorname{supp} \bar{f}$,

$$
\int_{X} f^{0}(y) e^{-C t|x-y|} d y=C^{-d} t^{-d} f_{C t}^{0}(x)
$$

with $f_{r}^{0}$ defined as in (1.20). This implies that

$$
f(t, x) \leq C \frac{t^{d}}{f_{C t}^{0}(x)}, \quad \forall x \in \operatorname{supp} \bar{f},
$$

and finally

$$
\tilde{F}(t) \geq-C+\int_{X} \bar{f} \log \frac{f_{C t}^{0}(x)}{t^{d}} \geq-C-C \log t,
$$

because of (1.20).

Step 2: Control on the dissipation term.

We compute the time derivative of $\tilde{F}$ :

$$
\begin{aligned}
\frac{d}{d t} \tilde{F}(t)= & \int_{X}\left(\frac{-\bar{f}(x)}{f(t, x)} \partial_{t} f(t, x)+\partial_{t} f(t, x)\right) M(x) d x \\
= & \int_{X}-\bar{f}(x)\left(a(x)-\int_{X} b(x, y) f(t, y) d y\right) M(x) d x \\
& +\int_{X} f(t, x)\left(a(x)-\int_{X} b(x, y) f(t, y) d y\right) M(x) d x
\end{aligned}
$$


Using the definition of the ESD (1.4), and in particular the fact that $a-b[\bar{f}]$ vanishes on the support of $\bar{f}$,

$$
\begin{aligned}
\frac{d}{d t} \tilde{F}(t)= & -\int_{X} \bar{f}(x)(b[\bar{f}]-b[f]) M(x) d x+\int_{X} f(t, x)(b[\bar{f}]-b[f]) M(x) d x \\
& +\int_{X} f(t, x)(a(x)-b[\bar{f}]) M(x) d x \\
= & -\int_{X^{2}} b(x, y)(\bar{f}(x)-f(t, x))(\bar{f}(y)-f(t, y)) M(x) d y d x \\
& +\int_{X} f(t, x)(a(x)-b[\bar{f}]) M(x) d x .
\end{aligned}
$$

Since $\tilde{F}$ is not bounded from below, it is not enough to show that $\tilde{F}$ is decreasing: we need to estimate $\frac{d}{d t} \tilde{F}$ from above. We then define a new functional $G$ :

$$
\begin{aligned}
G(t):= & -\int_{X^{2}} b(x, y)(\bar{f}(x)-f(t, x))(\bar{f}(y)-f(t, y)) M(x) d y d x \\
& +\int_{X} f(t, x)(a(x)-b[\bar{f}])(M(x)+N(x)) d x
\end{aligned}
$$

Notice that thanks to Assumption (1.18) and the bounds on $M$ and $N$ (1.17), $G$ controls $\frac{d}{d t} \tilde{F}(t)$ from above:

$$
\begin{aligned}
\frac{d}{d t} \tilde{F}(t) & \leq \inf _{x \in \mathbb{R}}\left[\frac{M(x)}{M(x)+N(x)}\right] G(t) \\
& \leq C G(t)
\end{aligned}
$$

The additional structure provided by (1.17) yields the last property that we need, namely that $G$ is non decreasing

$$
\begin{aligned}
& \frac{d}{d t} G(t)=-\int_{X^{2}} b(x, y)(f(t, x)-\bar{f}(x))(a(y)-b[f](y)) f(t, y) M(x) d x d y \\
& \left.\quad-\int_{X^{2}} b(x, y)(a(x)-b[f](x)) f(t, x)(f(t, y)-\bar{f}(y))\right) M(x) d x d y \\
& \quad+\int_{X}(a(x)-b[\bar{f}](x))(a(x)-b[f](x)) f(t, x)(M(x)+N(x)) d x \\
& =-\int_{X^{2}} b(x, y)(f(t, y)-\bar{f}(y))(a(x)-b[f](x)) f(t, x)(M(x)+N(x)) d x d y \\
& \quad+\int_{X}(a(x)-b[\bar{f}](x))(a(x)-b[f](x)) f(t, x)(M(x)+N(x)) d x .
\end{aligned}
$$


Hence

$$
\frac{d}{d t} G(t)=\int_{X}(a(x)-b[f](x))^{2} f(t, x)(M(x)+N(x)) d x \geq 0 .
$$

Step 3: Conclusion of the proof.

In this last part, we will combine the estimates obtained in the first and second step to conclude the proof. Thanks to (2.6),

$$
\begin{aligned}
C \frac{\log t+1}{t} & \geq-\frac{\tilde{F}(t)-\tilde{F}(0)}{t} \\
& =-\frac{1}{t} \int_{0}^{t} \frac{d}{d t} \tilde{F}(s) d s \\
& \geq-\max _{s \in[0, t]} \frac{d}{d t} \tilde{F}(s) .
\end{aligned}
$$

Then, using (2.8),

$$
\begin{aligned}
C \frac{\log t+1}{t} & \geq-C \max _{s \in[0, t]} G(s) \\
& \geq-C G(t),
\end{aligned}
$$

as $G$ is non decreasing. This estimate implies the convergence of $f(t, \cdot)$ to $\bar{f}$ w.r.t. the norm $\|\cdot\|_{b M}$, and estimates (1.21), (1.22), thus concluding the proof of the theorem.

\subsection{Proof of Corollary 3.1}

We prove the corollary in the case when $b_{1}$ satisfies (1.17) and (1.18). The case when $b_{1}$ satisfies (1.9) and $\bar{f}_{1} \in L \log L \cap L^{1}(X)$ is similar.

Let us consider $f$ the solution of (1.1) for $a_{1}, b_{1}$, corresponding to the following initial datum: $f^{0}=1$ on some large ball $B(0, R)$, and for $|x|>R$, $f^{0}(x)=|x|^{-d+1} / R^{d+1}$.

Therefore $f^{0} \in L^{1}(X) \cap L^{\infty}(X)$, and $f_{r}^{0}$ defined in (1.20) satisfies

$$
f_{r}^{0}(x) \geq \frac{r^{d}}{e} \int_{\left\{y ;|x-y| \leq \frac{1}{r}\right\}} f^{0}(y) d y \geq C|x|^{-d+1} .
$$

Then, $f^{0}$ satisfies (1.20) since

$$
\int_{X} \bar{f}_{1} \log |x|<\infty .
$$


Hence all the assumptions of Thm. 3 are satisfied and we know that $f(t, \cdot)$ converges to $\bar{f}_{1}$ in the sense

$$
\left\|f(t, \cdot)-\bar{f}_{1}\right\|_{b_{1} M} \rightarrow 0
$$

Denote now $\bar{f}_{2}^{R}=\bar{f}_{2} \mathbb{I}_{|x| \leq R / 2}$ and note that

$$
\int_{X}\left|\bar{f}_{2}-\bar{f}_{2}^{R}\right|=\varepsilon(R) \longrightarrow 0, \quad \text { as } R \rightarrow+\infty .
$$

We modify the functional $F$ of the proof of Thm 2 as follows:

$$
\check{F}(t)=\int_{X}\left[\bar{f}_{2}^{R}(x) \log \left(\frac{1}{f(t, x)}\right)+\left(f(t, x)-\bar{f}_{2}(x)\right)\right] M(x) d x .
$$

Using a regularization as in 2.2 , we compute the time derivative of $\check{F}$ :

$$
\begin{aligned}
\frac{d}{d t} \check{F}(t)= & \int_{X}\left(a_{1}(x)-b_{1}[f](t, x)\right)\left(f(t, x)-\bar{f}_{2}^{R}(x)\right) M(x) d x \\
= & \int_{X}\left(a_{2}(x)-b_{2}[t, f](x)\right)\left(f(t, x)-\bar{f}_{2}(x)\right) M(x) d x \\
+ & O\left[\varepsilon(R)+\left(\left\|a_{1}-a_{2}\right\|_{\infty}+\left\|b_{1}-b_{2}\right\|_{\infty}\|f\|_{M^{1}}\right)\left(\|f\|_{M^{1}}+\left\|\bar{f}_{2}\right\|_{M^{1}}\right)\right] \\
= & -\int_{X^{2}} b_{2}(x, y)\left(f(t, x)-\bar{f}_{2}(x)\right)\left(f(t, y)-\bar{f}_{2}(y)\right) M(x) d x d y \\
& +\int\left(a_{2}(x)-b_{2}\left[\bar{f}_{2}\right](x)\right) f(t, x) M(x) d x \\
& +O\left(\varepsilon(R)+\left\|a_{1}-a_{2}\right\|_{\infty}+\left\|b_{1}-b_{2}\right\|_{\infty}\right) .
\end{aligned}
$$

We thus conclude

$$
\begin{aligned}
\frac{d}{d t} \check{F}(t) \leq & \left.-\int_{X^{2}} b_{2}(x, y)\left(f(t, x)-\bar{f}_{2}(x)\right)\left(f(t, y)-\bar{f}_{2}(y)\right)\right) M(x) d x d y \\
& +O\left(\varepsilon(R)+\left\|a_{1}-a_{2}\right\|_{\infty}+\left\|b_{1}-b_{2}\right\|_{\infty}\right) .
\end{aligned}
$$

Define $I(t):=\int_{X^{2}} b_{2}(x, y)\left(f(t, x)-\bar{f}_{2}(x)\right)\left(f(t, y)-\bar{f}_{2}(y)\right) M(x) d x d y$. We estimate it as follows:

$$
\begin{aligned}
I(t) & =\int_{X^{2}} b_{1}(x, y)\left(f(t, x)-\bar{f}_{2}(x)\right)\left(f(t, y)-\bar{f}_{2}(y)\right) M(x) d x d y \\
& +\int_{X^{2}}\left(b_{2}(x, y)-b_{1}(x, y)\right)\left(f(t, x)-\bar{f}_{2}(x)\right)\left(f(t, y)-\bar{f}_{2}(y)\right) M(x) d x d y \\
& =\int_{X^{2}} b_{1}(x, y)\left(f(t, x)-\bar{f}_{2}(x)\right)\left(f(t, y)-\bar{f}_{2}(y)\right) M(x) d x d y \\
& \quad+O\left(\left\|b_{1}-b_{2}\right\|_{\infty}\right) .
\end{aligned}
$$


Proceeding further,

$$
\begin{aligned}
I(t)= & \int_{X^{2}} b_{1}(x, y)\left(f(t, x)-\bar{f}_{1}(x)\right)\left(f(t, y)-\bar{f}_{1}(y)\right) M(x) d x d y \\
& +\int_{X^{2}} b_{1}(x, y)\left(\bar{f}_{1}(x)-\bar{f}_{2}(x)\right)\left(\bar{f}_{1}(y)-\bar{f}_{2}(y)\right) M(x) d x d y \\
& +\int_{X^{2}} b_{1}(x, y)\left(f(t, x)-\bar{f}_{1}(x)\right)\left(\bar{f}_{1}(y)-\bar{f}_{2}(y)\right) M(x) d x d y \\
& +\int_{X^{2}} b_{1}(x, y)\left(\bar{f}_{1}(x)-\bar{f}_{2}(x)\right)\left(f(t, y)-\bar{f}_{1}(y)\right) M(x) d x d y \\
& +O\left(\left\|b_{1}-b_{2}\right\|_{\infty}\right) .
\end{aligned}
$$

Using the Cauchy-Schwarz inequality associated to the scalar product $\langle\cdot, \cdot\rangle_{b_{1} M}$ defined in Prop 1, we get

$$
\begin{aligned}
I(t)= & \left\|f(t, \cdot)-\bar{f}_{1}\right\|_{b_{1} M}^{2}+\left\|\bar{f}_{1}-\bar{f}_{2}\right\|_{b_{1} M}^{2}+2\left\|\bar{f}_{1}-\bar{f}_{2}\right\|_{b_{1} M}\left\|f(t, \cdot)-\bar{f}_{1}\right\|_{b_{1} M} \\
& +O\left(\left\|b_{1}-b_{2}\right\|_{\infty}\right) .
\end{aligned}
$$

As $f$ is the solution of (1.1) for $a_{1}, b_{1}$, Thm 3 applies, that is $\| f(t, \cdot)-$ $\bar{f}_{1} \|_{b_{1} M} \rightarrow 0$. Then,

$$
I(t)=\left\|\bar{f}_{1}-\bar{f}_{2}\right\|_{b_{1} M}^{2}+O\left(\left\|b_{1}-b_{2}\right\|_{\infty}\right)+o_{t \rightarrow \infty}(1) .
$$

Using this estimate in (2.9), we get:

$$
\frac{d}{d t} \check{F}(t) \leq-\left\|\bar{f}_{1}-\bar{f}_{2}\right\|_{b_{1} M}^{2}+O\left(\varepsilon(R)+\left\|a_{1}-a_{2}\right\|_{\infty}+\left\|b_{1}-b_{2}\right\|_{\infty}\right)+o_{t \rightarrow \infty}(1),
$$

so that

$$
\begin{aligned}
\check{F}(t) \leq & C-t\left(\left\|\bar{f}_{1}-\bar{f}_{2}\right\|_{b_{1} M}^{2}+O\left(\varepsilon(R)+\left\|a_{1}-a_{2}\right\|_{\infty}+\left\|b_{1}-b_{2}\right\|_{\infty}\right)\right) \\
& +o_{t \rightarrow \infty}(t) .
\end{aligned}
$$

To complete the proof, we notice that the estimates done in step 1 of the proof of Thm 3 can be reproduced, and since $\inf _{B(0, R)} f^{0}(x)>0$, we get, for any $x \in \operatorname{supp} \bar{f}_{2}^{R} \subset B(0, R)$,

$$
f(t, x) \leq C t^{d}
$$

Then, still similarly to the begining of step 1 of the proof of Thm 3, one obtains the following lower bound on $\check{F}$, similar to (2.6):

$$
\begin{aligned}
\check{F}(t) & \geq-C_{R}-\int \bar{f}_{2}^{R}(x) \log (f(t, x)) M(x) d x \\
& \geq-C_{R}-C_{R} \int \bar{f}_{2}^{R}(x) \log \left(t^{d}\right) M(x) d x \\
& \geq-C_{R}-C_{R} \log (t) .
\end{aligned}
$$


Combining the two inequalities (2.10) and (2.11), we get:

$$
\left\|\bar{f}_{1}-\bar{f}_{2}^{R}\right\|_{b_{1} M}^{2}+O\left(\left\|a_{1}-a_{2}\right\|_{\infty}+\left\|b_{1}-b_{2}\right\|_{\infty}\right)=o_{t \rightarrow+\infty}(1)+O(\varepsilon(R)),
$$

which provides the result by letting $t \rightarrow+\infty$ first and then $R \rightarrow+\infty$ :

$$
\left\|\bar{f}_{1}-\bar{f}_{2}\right\|_{b_{1} M}^{2} \leq O\left(\left\|a_{1}-a_{2}\right\|_{\infty}+\left\|b_{1}-b_{2}\right\|_{\infty}\right) .
$$

\section{References}

[1] M. Ackermann, M. Doebeli, Evolution of niche width and adaptive diversification. Evol. 58 (12), 2599-2612, (2004).

[2] G. Barles, B. Perthame, Concentrations and constrained HamiltonJacobi equations arising in adaptive dynamics. Recent developments in nonlinear partial differential equations, 57-68, Contemp. Math., 439, Amer. Math. Soc., Providence, RI, 2007.

[3] R. Bürger, The Mathematical Theory of Selection, Recombination and Mutation. Wiley, New-York, 2000.

[4] A. Calcina, S. Cuadrado, Small mutation rate and evolutionarily stable strategies in infinite dimensional adaptive dynamics. J. Math. Biol. 48, 135-159, (2004).

[5] N. Champagnat, R. Ferrière, G. Ben Arous, The canonical equation of adaptive dynamics: a mathematical view. Selection 2, 71-81, (2001).

[6] N. Champagnat, R. Ferrière, S. Méléard, From individual stochastic processes to macroscopic models in adaptive evolution. Stoch. Models 24, suppl.1, 2-44, (2008).

[7] L. Desvillettes, P.E. Jabin, S. Mischler, G. Raoul,On selection dynamics for continuous populations. Commun. Math. Sci, 6, no.3, 729-747 (2008).

[8] O. Diekmann, Beginner's guide to adaptive dynamics, 63, Banach Center Publications, 47-86 (2004).

[9] O. Diekmann, M. Gyllenberg and J.A.J. Metz, Steady states analysis of structured population models. Theor. Popul. Biol. 63, 309-338, (2003).

[10] O. Diekmann, P.E. Jabin, S. Mischler, B. Perthame, The dynamics of adaptation: An illuminating example and a Hamilton-Jacobi approach. Theor. Popul. Biol. 67, 257-271, (2005). 
[11] M. Doebeli, H.J. Blok, O. Leimar, U. Dieckmann. Multimodal pattern formation in phenotype distributions of sexual populations. Proc. $R$. Soc. B 274, 347-357, (2007).

[12] U. Dieckmann and R. Law, The dynamical theory of coevolution: A derivation from a stochastic ecological processes. J. Math. Biol. 34, 579-612, (1996).

[13] R. Ferrière, J.L. Bronstein, S. Rinaldi, R. Law and M. Gauduchon, Cheating and the evolutionary stability of mutualisms. Proc. Royal Soc. London Ser. B 269, 773-780, (2002).

[14] S. Genieys, V. Volpert, P. Auger. Pattern and waves for a model in population dynamics with nonlocal consumption of resources. Math. Model. Nat. Phenom. 1, 65-82, (2006).

[15] S.A.H. Geritz, Resident-invader dynamics and the coexistence of similar strategies. J. Math. Biol. 50, 67-82, (2005).

[16] S.A.H. Geritz, É. Kisdi, G. Meszéna and J.A.J. Metz, Evolutionarily singular strategies and the adaptive growth and branching of the evolutionary tree. Evol. Ecol. 12, 35-57, (1998).

[17] Gyllenberg M., Meszéna G., On the impossibility of coexistence of infinitely many strategies, J. Math. Biol. 50, 133-160, (2005).

[18] B. Perthame, Transport Equation in Biology, Frontiers in Mathematics series, Birkhäuser, 2005.

[19] Perthame, S. Genieys, Concentration in the nonlocal Fisher equation: the Hamilton-Jacobi limit. Math. Model. Nat. Phenom. 2, no. 4, 135151, (2007).

[20] G. Raoul, Local stability of Evolutionary Attractors for continuous structured populations, preprint CMLA, ENS Cachan, no.2009-01 (2009).

[21] G. Raoul, Long time evolution of populations under selection and rare mutations, in progress.

[22] J. Roughgarden, Theory of population genetics and evolutionary ecology. New York, NY: Macmillan 1979.

[23] A. Sasaki, Clumped distribution by neighborhood competition. J. Theor. Biol. 186, 304-329, (1997). 
[24] A. Sasaki, S. Ellner, The evolutionarily stable phenotype distribution in a random environment. Evolution 49, 337-350, (1995). 